\title{
Supplemental material for: Characterization of the short-range couplings in excitation energy transfer
}

\author{
Chao-Ping Hsu ${ }^{1 *}$ Zhi-Qiang You $^{1,2}$ and Hung-Cheng Chen ${ }^{1}$ \\ ${ }^{1}$ Institute of Chemistry, Academia Sinica, \\ 128 Section 2 Academia Road, Nankang, Taipei 115, Taiwan \\ ${ }^{2}$ Department of Chemistry, National Tsing Hua University, \\ 101, Section 2, Kuang-Fu Road, Hsinchu, Taiwan 30013
}

October 7, 2007

The full citation for Ref. 38 of the main text is:

(38) Shao, Y.; Fusti-Molnar, L.; Jung, Y.; Kussmann, J.; Ochsenfeld, C.; Brown, S. T.; Gilbert, A. T. B.; Slipchenko, L. V.; Levchenko, S. V.; O’Neill, D. P.; Distasio Jr., R. A.; Lochan, R. C.; Wang, T.; Beran, G. J. O.; Besley, N. A.; Herbert, J. M.; Lin, C. Y.; van Voorhis, T., Chien, S. H.; Sodt, A.; Steele, R. P.; Rassolov, V. A.; Maslen, P. E.; Korambath, P. P.; Adamson, R. D.; Austin, B.; Baker, J.; Byrd, E. F. C.; Dachsel, H.; Doerksen, R. J.; Dreuw, A.; Dunietz, B. D.; Dutoi, A. D.; Furlani, T. R.; Gwaltney, S. R.; Heyden, A.; Hirata, S.; Hsu, C.-P.; Kedziora, G.; Khalliulin, R. Z.; Klunzinger, P.; Lee, A. M.; Lee, M. S.; Liang, W.; Lotan, I.; Nair, N.; Peters, B.; Proynov, E. I.; Pieniazek, P. A.; Rhee, Y. M.; Ritchie, J.; Rosta, E.; Sherrill, C. D.; Simmonett, A. C.; Subotnik, J. E.; Woodcock III, H. L.; Zhang, W.; Bell, A. T.; Chakraborty, A. K.; Chipman, D. M.; Keil, F. J.; Warshel, A.; Hehre, W. J.; Schaefer III, H. F.; Kong, J.; Krylov, A. I.; Gill, P. M. W. \& Head-Gordon, M. Phys. Chem. Chem. Phys. 2006, 8, 3172.

The structures of molecules studied in the present work are included in the following. The stacked naphthelenes are composed a molecules optimized at its singlet ground state and another at its triplet state. Their geometries are listed as follows:

$\begin{array}{crrc}\text { Singlet } & \text { Optimized / B3LYP/DZ(d) } & \mathrm{E}= & -385.917487967 \mathrm{~d} 2 \mathrm{~h} \\ \mathrm{C} & -1.247231 & -1.408472 & 0.000000 \\ \text { C } & -2.442246 & -0.711801 & 0.000000 \\ \text { C } & -2.442246 & 0.711801 & 0.000000 \\ \text { C } & -1.247231 & 1.408472 & 0.000000 \\ \text { C } & 0.000000 & 0.717709 & 0.000000 \\ \text { C } & 0.000000 & -0.717709 & 0.000000\end{array}$

*Corresponding author. Fax: 886-2-2783-1237. Email: cherri@sinica.edu.tw 


$\begin{array}{lrrr}\mathrm{C} & 1.247231 & -1.408472 & 0.000000 \\ \mathrm{C} & 2.442246 & -0.711801 & 0.000000 \\ \mathrm{C} & 2.442246 & 0.711801 & 0.000000 \\ \mathrm{C} & 1.247231 & 1.408472 & 0.000000 \\ \mathrm{H} & -1.245160 & -2.498925 & 0.000000 \\ \mathrm{H} & -3.389646 & -1.249013 & 0.000000 \\ \mathrm{H} & -3.389646 & 1.249013 & 0.000000 \\ \mathrm{H} & -1.245160 & 2.498925 & 0.000000 \\ \mathrm{H} & 1.245160 & -2.498925 & 0.000000 \\ \mathrm{H} & 3.389646 & -1.249013 & 0.000000 \\ \mathrm{H} & 3.389646 & 1.249013 & 0.000000 \\ \mathrm{H} & 1.245160 & 2.498925 & 0.000000\end{array}$

$\begin{array}{crrc}\text { Triplet } & \text { Optimized / B3LYP/DZ(d) } E= & -385.819586235 \mathrm{~d} 2 \mathrm{~h} \\ \mathrm{C} & -1.240990 & -1.407165 & 0.000000 \\ \mathrm{C} & -2.495729 & -0.685584 & 0.000000 \\ \mathrm{C} & -2.495729 & 0.685584 & 0.000000 \\ \mathrm{C} & -1.240990 & 1.407165 & 0.000000 \\ \mathrm{C} & 0.000000 & 0.725834 & 0.000000 \\ \mathrm{C} & 0.000000 & -0.725834 & 0.000000 \\ \mathrm{C} & 1.240990 & -1.407165 & 0.000000 \\ \mathrm{C} & 2.495729 & -0.685584 & 0.000000 \\ \mathrm{C} & 2.495729 & 0.685584 & 0.000000 \\ \mathrm{C} & 1.240990 & 1.407165 & 0.000000 \\ \mathrm{H} & -1.248476 & -2.496836 & 0.000000 \\ \mathrm{H} & -3.431212 & -1.242408 & 0.000000 \\ \mathrm{H} & -3.431212 & 1.242408 & 0.000000 \\ \mathrm{H} & -1.248476 & 2.496836 & 0.000000 \\ \mathrm{H} & 1.248476 & -2.496836 & 0.000000 \\ \mathrm{H} & 3.431212 & -1.242408 & 0.000000 \\ \mathrm{H} & 3.431212 & 1.242408 & 0.000000 \\ \mathrm{H} & 1.248476 & 2.496836 & 0.000000\end{array}$

The structures of polynorbornyl-linked naphthalene or anthracene dimers, and their truncated models are:

$\begin{array}{crrr}\text { DN2_Full } & & & \\ \text { C } & -1.223757 & 0.715452 & 0.975853 \\ \text { C } & -1.223757 & -0.715452 & 0.975853 \\ \text { C } & -2.203771 & 1.421820 & 0.337248 \\ \text { C } & -3.254026 & 0.718297 & -0.330296 \\ \text { C } & -3.254026 & -0.718297 & -0.330296 \\ \text { C } & -2.203771 & -1.421820 & 0.337248 \\ \text { C } & -4.311401 & 1.399544 & -0.985917\end{array}$




$\begin{array}{lrrr}\mathrm{C} & -5.325540 & 0.707011 & -1.612563 \\ \mathrm{C} & -5.325540 & -0.707011 & -1.612563 \\ \mathrm{C} & -4.311401 & -1.399544 & -0.985917 \\ \mathrm{C} & 0.000000 & 1.144740 & 1.785077 \\ \mathrm{C} & 0.000000 & -1.144740 & 1.785077 \\ \mathrm{C} & 0.000000 & 0.000000 & 2.852036 \\ \mathrm{H} & -2.214469 & -2.510147 & 0.337377 \\ \mathrm{H} & -2.214469 & 2.510147 & 0.337377 \\ \mathrm{H} & -4.310241 & 2.487610 & -0.983575 \\ \mathrm{H} & -6.130604 & 1.245467 & -2.104777 \\ \mathrm{H} & -6.130604 & -1.245467 & -2.104777 \\ \mathrm{H} & -4.310241 & -2.487610 & -0.983575 \\ \mathrm{H} & 0.000000 & 2.175219 & 2.144489 \\ \mathrm{H} & 0.000000 & -2.175219 & 2.144489 \\ \mathrm{H} & -0.897694 & 0.000000 & 3.478980 \\ \mathrm{H} & 0.897694 & 0.000000 & 3.478980 \\ \mathrm{C} & 1.223757 & 0.715452 & 0.975853 \\ \mathrm{C} & 1.223757 & -0.715452 & 0.975853 \\ \mathrm{C} & 2.203771 & -1.421820 & 0.337248 \\ \mathrm{C} & 2.203771 & 1.421820 & 0.337248 \\ \mathrm{C} & 3.254026 & -0.718297 & -0.330296 \\ \mathrm{C} & 3.254026 & 0.718297 & -0.330296 \\ \mathrm{C} & 4.311401 & -1.399544 & -0.985917 \\ \mathrm{C} & 4.311401 & 1.399544 & -0.985917 \\ \mathrm{C} & 5.325540 & -0.707011 & -1.612563 \\ \mathrm{C} & 5.325540 & 0.707011 & -1.612563 \\ \mathrm{H} & 2.214469 & -2.510147 & 0.337377 \\ \mathrm{H} & 2.214469 & 2.510147 & 0.337377 \\ \mathrm{H} & 4.310241 & -2.487610 & -0.983575 \\ \mathrm{H} & 4.310241 & 2.487610 & -0.983575 \\ & 6.130604 & 1.245467 & -2.104777 \\ \mathrm{H} & 6.130604 & -1.245467 & -2.104777\end{array}$

DN2 NO-NO

$\begin{array}{lrrr}\text { C } & 2.203923 & -1.421938 & 0.660551 \\ \text { C } & 3.254076 & -0.718671 & -0.007423 \\ \text { C } & 3.254076 & 0.718671 & -0.007423 \\ \text { C } & 2.203923 & 1.421938 & 0.660551 \\ \text { C } & 4.311537 & -1.400167 & -0.662647 \\ \text { C } & 5.325576 & -0.707871 & -1.289716 \\ \text { C } & 5.325576 & 0.707871 & -1.289716 \\ \text { C } & 4.311537 & 1.400167 & -0.662647 \\ \text { C } & 1.223890 & 0.715250 & 1.297910\end{array}$




$\begin{array}{lrrr}\mathrm{C} & 1.223890 & -0.715250 & 1.297910 \\ \mathrm{H} & 0.484616 & 1.340368 & 1.790893 \\ \mathrm{H} & 0.484616 & -1.340368 & 1.790893 \\ \mathrm{H} & 2.214086 & 2.510030 & 0.658329 \\ \mathrm{H} & 2.214086 & -2.510030 & 0.658329 \\ \mathrm{H} & 4.310525 & -2.488231 & -0.659654 \\ \mathrm{H} & 6.130709 & -1.246512 & -1.781615 \\ \mathrm{H} & 6.130709 & 1.246512 & -1.781615 \\ \mathrm{H} & 4.310525 & 2.488231 & -0.659654 \\ \mathrm{C} & -2.203922 & 1.421938 & 0.660551 \\ \mathrm{C} & -2.203923 & -1.421938 & 0.660551 \\ \mathrm{C} & -3.254076 & 0.718671 & -0.007423 \\ \mathrm{C} & -3.254076 & -0.718671 & -0.007423 \\ \mathrm{C} & -4.311537 & 1.400167 & -0.662647 \\ \mathrm{C} & -4.311537 & -1.400167 & -0.662647 \\ \mathrm{C} & -5.325576 & 0.707871 & -1.289716 \\ \mathrm{C} & -5.325576 & -0.707871 & -1.289716 \\ \mathrm{C} & -1.223890 & 0.715250 & 1.297910 \\ \mathrm{C} & -1.223890 & -0.715250 & 1.297910 \\ \mathrm{H} & -2.214086 & 2.510030 & 0.658329 \\ \mathrm{H} & -2.214086 & -2.510030 & 0.658329 \\ \mathrm{H} & -4.310525 & 2.488231 & -0.659654 \\ \mathrm{H} & -4.310525 & -2.488231 & -0.659654 \\ \mathrm{H} & -6.130709 & -1.246512 & -1.781615 \\ \mathrm{H} & -6.130709 & 1.246512 & -1.781615 \\ \mathrm{H} & -0.484616 & 1.340368 & 1.790893 \\ \mathrm{H} & -0.484616 & -1.340368 & 1.790893\end{array}$

DN4 Full

$\begin{array}{lrrr}\text { C } & -2.412290 & 0.716817 & 0.885068 \\ \text { C } & -2.412290 & -0.716817 & 0.885068 \\ \text { C } & -3.232296 & 1.421931 & 0.047291 \\ \text { C } & -4.112571 & 0.718149 & -0.830674 \\ \text { C } & -4.112571 & -0.718149 & -0.830674 \\ \text { C } & -3.232296 & -1.421931 & 0.047291 \\ \text { C } & -4.994924 & 1.400186 & -1.707861 \\ \text { C } & -5.837828 & 0.707146 & -2.549997 \\ \text { C } & -5.837828 & -0.707146 & -2.549997 \\ \text { C } & -4.994924 & -1.400186 & -1.707861 \\ \text { C } & -1.363271 & 1.140110 & 1.905186 \\ \text { C } & -1.363271 & -1.140110 & 1.905186 \\ \text { C } & -1.578004 & 0.000000 & 2.937027 \\ \text { C } & 0.000000 & 0.789430 & 1.216935\end{array}$




\begin{tabular}{|c|c|c|c|}
\hline $\mathrm{C}$ & 0.000000 & -0.789430 & 1.216935 \\
\hline $\mathrm{H}$ & -3.238495 & -2.510457 & 0.038649 \\
\hline $\mathrm{H}$ & -3.238495 & 2.510457 & 0.038649 \\
\hline $\mathrm{H}$ & -4.993546 & 2.488180 & -1.705843 \\
\hline $\mathrm{H}$ & -6.505819 & 1.245197 & -3.217670 \\
\hline $\mathrm{H}$ & -6.505819 & -1.245197 & -3.217670 \\
\hline $\mathrm{H}$ & -4.993546 & -2.488180 & -1.705843 \\
\hline $\mathrm{H}$ & -1.444218 & 2.167533 & 2.269665 \\
\hline $\mathrm{H}$ & -1.444218 & -2.167533 & 2.269665 \\
\hline $\mathrm{H}$ & -2.600153 & 0.000000 & 3.329234 \\
\hline $\mathrm{H}$ & 0.000000 & 1.205238 & 0.206130 \\
\hline $\mathrm{H}$ & 0.000000 & -1.205238 & 0.206130 \\
\hline $\mathrm{H}$ & -0.892391 & 0.000000 & 3.783085 \\
\hline $\mathrm{C}$ & 1.363271 & -1.140110 & 1.905186 \\
\hline C & 1.363271 & 1.140110 & 1.905186 \\
\hline $\mathrm{C}$ & 1.578004 & 0.000000 & 2.937027 \\
\hline $\mathrm{H}$ & 1.444218 & -2.167533 & 2.269665 \\
\hline $\mathrm{H}$ & 1.444218 & 2.167533 & 2.269665 \\
\hline $\mathrm{H}$ & 2.600153 & 0.000000 & 3.329234 \\
\hline $\mathrm{H}$ & 0.892391 & 0.000000 & 3.783085 \\
\hline $\mathrm{C}$ & 2.412290 & 0.716817 & 0.885068 \\
\hline $\mathrm{C}$ & 2.412290 & -0.716817 & 0.885068 \\
\hline $\mathrm{C}$ & 3.232296 & -1.421931 & 0.047291 \\
\hline C & 3.232296 & 1.421931 & 0.047291 \\
\hline $\mathrm{C}$ & 4.112571 & -0.718149 & -0.830674 \\
\hline $\mathrm{C}$ & 4.112571 & 0.718149 & -0.830674 \\
\hline $\mathrm{C}$ & 4.994924 & -1.400186 & -1.707861 \\
\hline C & 4.994924 & 1.400186 & -1.707861 \\
\hline $\mathrm{C}$ & 5.837828 & -0.707146 & -2.549997 \\
\hline $\mathrm{C}$ & 5.837828 & 0.707146 & -2.549997 \\
\hline $\mathrm{H}$ & 3.238495 & -2.510457 & 0.038649 \\
\hline $\mathrm{H}$ & 3.238495 & 2.510457 & 0.038649 \\
\hline $\mathrm{H}$ & 4.993546 & -2.488180 & -1.705843 \\
\hline $\mathrm{H}$ & 4.993546 & 2.488180 & -1.705843 \\
\hline $\mathrm{H}$ & 6.505819 & 1.245197 & -3.217670 \\
\hline $\mathrm{H}$ & 6.505819 & -1.245197 & -3.217670 \\
\hline
\end{tabular}

DN4 N1-N1

$\begin{array}{lrrr}\text { C } & -2.412282 & 0.716817 & 0.940424 \\ \text { C } & -2.412282 & -0.716817 & 0.940424 \\ \text { C } & -3.232292 & 1.421931 & 0.102650 \\ \text { C } & -4.112572 & 0.718149 & -0.775311 \\ \text { C } & -4.112572 & -0.718149 & -0.775311\end{array}$




\begin{tabular}{|c|c|c|c|}
\hline C & -3.232292 & -1.421931 & 0.102650 \\
\hline $\mathrm{C}$ & -4.994932 & 1.400185 & -1.652489 \\
\hline C & -5.837846 & 0.707145 & -2.494618 \\
\hline C & -5.837846 & -0.707145 & -2.494618 \\
\hline $\mathrm{C}$ & -4.994932 & -1.400185 & -1.652489 \\
\hline C & -1.577997 & 0.000000 & 2.992381 \\
\hline C & -1.363275 & -1.140110 & 1.960555 \\
\hline$C$ & -1.363275 & 1.140110 & 1.960555 \\
\hline $\mathrm{H}$ & -0.552600 & -1.030341 & 1.325529 \\
\hline $\mathrm{H}$ & -0.552600 & 1.030341 & 1.325529 \\
\hline $\mathrm{H}$ & -3.238493 & -2.510457 & 0.094011 \\
\hline $\mathrm{H}$ & -3.238493 & 2.510457 & 0.094011 \\
\hline $\mathrm{H}$ & -4.993556 & 2.488180 & -1.650473 \\
\hline $\mathrm{H}$ & -6.505842 & 1.245199 & -3.162284 \\
\hline $\mathrm{H}$ & -6.505842 & -1.245199 & -3.162284 \\
\hline $\mathrm{H}$ & -4.993556 & -2.488180 & -1.650473 \\
\hline $\mathrm{H}$ & -2.600143 & 0.000000 & 3.384596 \\
\hline $\mathrm{H}$ & -0.892377 & 0.000000 & 3.838432 \\
\hline $\mathrm{H}$ & -1.442509 & 2.145767 & 2.317313 \\
\hline $\mathrm{H}$ & -1.442509 & -2.145767 & 2.317313 \\
\hline $\mathrm{C}$ & 1.577997 & 0.000000 & 2.992381 \\
\hline $\mathrm{C}$ & 1.363275 & 1.140110 & 1.960555 \\
\hline $\mathrm{C}$ & 1.363275 & -1.140110 & 1.960555 \\
\hline$C$ & 2.412282 & 0.716817 & 0.940424 \\
\hline $\mathrm{C}$ & 2.412282 & -0.716817 & 0.940424 \\
\hline $\mathrm{C}$ & 3.232292 & -1.421931 & 0.102650 \\
\hline C & 3.232292 & 1.421931 & 0.102650 \\
\hline $\mathrm{C}$ & 4.112572 & -0.718149 & -0.775311 \\
\hline C & 4.112572 & 0.718149 & -0.775311 \\
\hline $\mathrm{C}$ & 4.994932 & -1.400185 & -1.652489 \\
\hline $\mathrm{C}$ & 4.994932 & 1.400185 & -1.652489 \\
\hline $\mathrm{C}$ & 5.837846 & -0.707145 & -2.494618 \\
\hline C & 5.837846 & 0.707145 & -2.494618 \\
\hline $\mathrm{H}$ & 3.238493 & -2.510457 & 0.094011 \\
\hline $\mathrm{H}$ & 3.238493 & 2.510457 & 0.094011 \\
\hline $\mathrm{H}$ & 4.993556 & -2.488180 & -1.650473 \\
\hline $\mathrm{H}$ & 4.993556 & 2.488180 & -1.650473 \\
\hline $\mathrm{H}$ & 6.505842 & 1.245199 & -3.162284 \\
\hline $\mathrm{H}$ & 6.505842 & -1.245199 & -3.162284 \\
\hline $\mathrm{H}$ & 0.552600 & -1.030341 & 1.325529 \\
\hline$\Pi$ & 0.552600 & 1.030341 & 1.325529 \\
\hline $\mathrm{H}$ & 2.600143 & 0.000000 & 3.384596 \\
\hline $\mathrm{H}$ & 0.892377 & 0.000000 & 3.838432 \\
\hline
\end{tabular}




$\begin{array}{rrrr}\mathrm{H} & 1.442509 & -2.145767 & 2.317313 \\ \mathrm{H} & 1.442509 & 2.145767 & 2.317313\end{array}$

DN4 N1-NO

$\begin{array}{lrrr}\mathrm{C} & -2.272722 & -1.182665 & 0.716817 \\ \mathrm{C} & -2.272722 & -1.182665 & -0.716817 \\ \mathrm{C} & -3.058954 & -0.313114 & 1.421931 \\ \mathrm{C} & -3.903821 & 0.598976 & 0.718149 \\ \mathrm{C} & -3.903821 & 0.598976 & -0.718149 \\ \mathrm{C} & -3.058954 & -0.313114 & -1.421931 \\ \mathrm{C} & -4.750797 & 1.510367 & 1.400185 \\ \mathrm{C} & -5.559744 & 2.385176 & 0.707145 \\ \mathrm{C} & -5.559744 & 2.385176 & -0.707145 \\ \mathrm{C} & -4.750797 & 1.510367 & -1.400185 \\ \mathrm{C} & -1.264873 & -2.243474 & 1.140110 \\ \mathrm{C} & -1.264873 & -2.243474 & -1.140110 \\ \mathrm{C} & -1.520248 & -3.266014 & 0.000000 \\ \mathrm{H} & -3.064810 & -0.304236 & -2.510457 \\ \mathrm{H} & -3.064810 & -0.304236 & 2.510457 \\ \mathrm{H} & -4.749501 & 1.508299 & 2.488180 \\ \mathrm{H} & -6.200809 & 3.078740 & 1.245199 \\ \mathrm{H} & -6.200809 & 3.078740 & -1.245199 \\ \mathrm{H} & -4.749501 & 1.508299 & -2.488180 \\ \mathrm{H} & -1.360174 & -2.604467 & 2.167533 \\ \mathrm{H} & -1.360174 & -2.604467 & -2.167533 \\ \mathrm{H} & -2.229783 & 1.113304 & 2.488180 \\ \mathrm{H} & & & \end{array}$




$\begin{array}{lrrr}\mathrm{H} & 6.800654 & 2.564146 & 1.245199 \\ \mathrm{H} & 6.800654 & 2.564146 & -1.245199 \\ \mathrm{H} & 1.783432 & -2.060375 & 1.014159 \\ \mathrm{H} & 1.783432 & -2.060375 & -1.014159\end{array}$

DN4 NO-NO

\begin{tabular}{|c|c|c|c|}
\hline C & -2.412282 & 0.716817 & 1.714730 \\
\hline C & -2.412282 & -0.716817 & 1.714730 \\
\hline $\mathrm{C}$ & -3.232292 & 1.421931 & 0.876957 \\
\hline C & -4.112572 & 0.718149 & -0.001004 \\
\hline C & -4.112572 & -0.718149 & -0.001004 \\
\hline $\mathrm{C}$ & -3.232292 & -1.421931 & 0.876957 \\
\hline $\mathrm{C}$ & -4.994932 & 1.400185 & -0.878182 \\
\hline C & -5.837846 & 0.707145 & -1.720311 \\
\hline $\mathrm{C}$ & -5.837846 & -0.707145 & -1.720311 \\
\hline $\mathrm{C}$ & -4.994932 & -1.400185 & -0.878182 \\
\hline $\mathrm{H}$ & -3.238493 & -2.510457 & 0.868317 \\
\hline $\mathrm{H}$ & -3.238493 & 2.510457 & 0.868317 \\
\hline $\mathrm{H}$ & -4.993556 & 2.488180 & -0.876166 \\
\hline $\mathrm{H}$ & -6.505842 & 1.245199 & -2.387977 \\
\hline $\mathrm{H}$ & -6.505842 & -1.245199 & -2.387977 \\
\hline $\mathrm{H}$ & -4.993556 & -2.488180 & -0.876166 \\
\hline $\mathrm{H}$ & -1.663720 & 1.018875 & 2.442686 \\
\hline $\mathrm{H}$ & -1.663720 & -1.018875 & 2.442686 \\
\hline $\mathrm{C}$ & 2.412282 & 0.716817 & 1.714730 \\
\hline $\mathrm{C}$ & 2.412282 & -0.716817 & 1.714730 \\
\hline $\mathrm{C}$ & 3.232292 & -1.421931 & 0.876957 \\
\hline $\mathrm{C}$ & 3.232292 & 1.421931 & 0.876957 \\
\hline $\mathrm{C}$ & 4.112572 & -0.718149 & -0.001004 \\
\hline $\mathrm{C}$ & 4.112572 & 0.718149 & -0.001004 \\
\hline $\mathrm{C}$ & 4.994932 & -1.400185 & -0.878182 \\
\hline $\mathrm{C}$ & 4.994932 & 1.400185 & -0.878182 \\
\hline $\mathrm{C}$ & 5.837846 & -0.707145 & -1.720311 \\
\hline $\mathrm{C}$ & 5.837846 & 0.707145 & -1.720311 \\
\hline $\mathrm{H}$ & 3.238493 & -2.510457 & 0.868317 \\
\hline $\mathrm{H}$ & 3.238493 & 2.510457 & 0.868317 \\
\hline $\mathrm{H}$ & 4.993556 & -2.488180 & -0.876166 \\
\hline $\mathrm{H}$ & 4.993556 & 2.488180 & -0.876166 \\
\hline $\mathrm{H}$ & 6.505842 & 1.245199 & -2.387977 \\
\hline $\mathrm{H}$ & 6.505842 & -1.245199 & -2.387977 \\
\hline $\mathrm{H}$ & 1.663720 & 1.018875 & 2.442686 \\
\hline $\mathrm{H}$ & 1.663720 & -1.018875 & 2.44268 \\
\hline
\end{tabular}

DN6 Full 


\begin{tabular}{|c|c|c|c|}
\hline $\mathrm{C}$ & -3.660077 & 0.716456 & 0.439811 \\
\hline C & -3.660077 & -0.716456 & 0.439811 \\
\hline C & -4.450508 & 1.421885 & -0.426583 \\
\hline$C$ & -5.301506 & 0.718348 & -1.332174 \\
\hline $\mathrm{C}$ & -5.301506 & -0.718348 & -1.332174 \\
\hline $\mathrm{C}$ & -4.450508 & -1.421885 & -0.426583 \\
\hline $\mathrm{C}$ & -6.159816 & 1.400232 & -2.233609 \\
\hline$C$ & -6.980289 & 0.707306 & -3.097327 \\
\hline$C$ & -6.980289 & -0.707306 & -3.097327 \\
\hline C & -6.159816 & -1.400232 & -2.233609 \\
\hline$c$ & -2.660994 & 1.140683 & 1.503350 \\
\hline $\mathrm{C}$ & -2.660994 & -1.140683 & 1.503350 \\
\hline $\mathrm{C}$ & -2.891767 & 0.000000 & 2.529292 \\
\hline C & -1.269044 & 0.784904 & 0.899488 \\
\hline$C$ & -1.269044 & -0.784904 & 0.899488 \\
\hline $\mathrm{H}$ & -4.458005 & -2.510402 & -0.433231 \\
\hline $\mathrm{H}$ & -4.458005 & 2.510402 & -0.433231 \\
\hline $\mathrm{H}$ & -6.159100 & 2.488236 & -2.231015 \\
\hline $\mathrm{H}$ & -7.630370 & 1.245278 & -3.782494 \\
\hline $\mathrm{H}$ & -7.630370 & -1.245278 & -3.782494 \\
\hline $\mathrm{H}$ & -6.159100 & -2.488236 & -2.231015 \\
\hline $\mathrm{H}$ & -2.763273 & 2.166402 & 1.865968 \\
\hline $\mathrm{H}$ & -2.763273 & -2.166402 & 1.865968 \\
\hline $\mathrm{H}$ & -3.911937 & 0.000000 & 2.926100 \\
\hline $\mathrm{H}$ & -1.128869 & 1.282664 & -0.065483 \\
\hline $\mathrm{H}$ & -1.128869 & -1.282664 & -0.065483 \\
\hline $\mathrm{H}$ & -2.190679 & 0.000000 & 3.365850 \\
\hline $\mathrm{C}$ & 0.000000 & -0.807272 & 1.812768 \\
\hline $\mathrm{C}$ & 0.000000 & 0.807272 & 1.812768 \\
\hline $\mathrm{C}$ & 0.000000 & -1.757730 & 2.999406 \\
\hline $\mathrm{C}$ & 0.000000 & 1.757730 & 2.999406 \\
\hline $\mathrm{C}$ & 2.660994 & -1.140683 & 1.503350 \\
\hline $\mathrm{C}$ & 2.660994 & 1.140683 & 1.503350 \\
\hline $\mathrm{C}$ & 2.891767 & 0.000000 & 2.529292 \\
\hline $\mathrm{C}$ & 1.269044 & 0.784904 & 0.899488 \\
\hline $\mathrm{C}$ & 1.269044 & -0.784904 & 0.899488 \\
\hline $\mathrm{H}$ & 0.881331 & -1.634661 & 3.636373 \\
\hline $\mathrm{H}$ & 0.000000 & -2.796957 & 2.643524 \\
\hline $\mathrm{H}$ & -0.881331 & -1.634661 & 3.636373 \\
\hline $\mathrm{H}$ & 0.881331 & 1.634661 & 3.636373 \\
\hline $\mathrm{H}$ & -0.881331 & 1.634661 & 3.636373 \\
\hline $\mathrm{H}$ & 0.000000 & 2.796957 & 2.643524 \\
\hline $\mathrm{H}$ & 2.763273 & -2.166402 & 1.865968 \\
\hline
\end{tabular}




$\begin{array}{lrrr}\mathrm{H} & 2.763273 & 2.166402 & 1.865968 \\ \mathrm{H} & 3.911937 & 0.000000 & 2.926100 \\ \mathrm{H} & 2.190679 & 0.000000 & 3.365850 \\ \mathrm{H} & 1.128869 & 1.282664 & -0.065483 \\ \mathrm{H} & 1.128869 & -1.282664 & -0.065483 \\ \mathrm{C} & 3.660077 & 0.716456 & 0.439811 \\ \mathrm{C} & 3.660077 & -0.716456 & 0.439811 \\ \mathrm{C} & 4.450508 & -1.421885 & -0.426583 \\ \mathrm{C} & 4.450508 & 1.421885 & -0.426583 \\ \mathrm{C} & 5.301506 & -0.718348 & -1.332174 \\ \mathrm{C} & 5.301506 & 0.718348 & -1.332174 \\ \mathrm{C} & 6.159816 & -1.400232 & -2.233609 \\ \mathrm{C} & 6.159816 & 1.400232 & -2.233609 \\ \mathrm{C} & 6.980289 & -0.707306 & -3.097327 \\ \mathrm{C} & 6.980289 & 0.707306 & -3.097327 \\ \mathrm{H} & 4.458005 & -2.510402 & -0.433231 \\ \mathrm{H} & 4.458005 & 2.510402 & -0.433231 \\ \mathrm{H} & 6.159100 & -2.488236 & -2.231015 \\ \mathrm{H} & 6.159100 & 2.488236 & -2.231015 \\ \mathrm{H} & 7.630370 & 1.245278 & -3.782494 \\ \mathrm{H} & 7.630370 & -1.245278 & -3.782494\end{array}$

DN6 N2-N2

$\begin{array}{lrrr}\text { C } & -3.660077 & 0.716456 & 0.780407 \\ \text { C } & -3.660077 & -0.716456 & 0.780408 \\ \text { C } & -4.450508 & 1.421885 & -0.085987 \\ \text { C } & -5.301506 & 0.718348 & -0.991578 \\ \text { C } & -5.301506 & -0.718348 & -0.991577 \\ \text { C } & -4.450508 & -1.421885 & -0.085986 \\ \text { C } & -6.159816 & 1.400232 & -1.893013 \\ \text { C } & -6.980289 & 0.707306 & -2.756731 \\ \text { C } & -6.980289 & -0.707306 & -2.756730 \\ \text { C } & -6.159816 & -1.400232 & -1.893012 \\ \text { C } & -2.660994 & 1.140683 & 1.843946 \\ \text { C } & -2.660994 & -1.140683 & 1.843947 \\ \text { C } & -2.891767 & 0.000000 & 2.869888 \\ \text { C } & -1.269044 & 0.784904 & 1.240084 \\ \text { C } & -1.269044 & -0.784904 & 1.240085 \\ \text { H } & -0.668451 & -1.211913 & 2.002390 \\ \text { H } & -0.668451 & 1.211913 & 2.002389 \\ \text { H } & -1.132044 & 1.271389 & 0.296970 \\ \text { H } & -1.132044 & -1.271389 & 0.296971 \\ \text { H } & -4.458005 & -2.510402 & -0.092634\end{array}$




\begin{tabular}{|c|c|c|c|}
\hline $\mathrm{H}$ & -4.458005 & 2.510402 & -0.092635 \\
\hline $\mathrm{H}$ & -6.159100 & 2.488236 & -1.890419 \\
\hline $\mathrm{H}$ & -7.630370 & 1.245278 & -3.441898 \\
\hline $\mathrm{H}$ & -7.630370 & -1.245278 & -3.441897 \\
\hline $\mathrm{H}$ & -6.159100 & -2.488236 & -1.890418 \\
\hline $\mathrm{H}$ & -2.763273 & 2.166402 & 2.206564 \\
\hline $\mathrm{H}$ & -2.763273 & -2.166402 & 2.206565 \\
\hline $\mathrm{H}$ & -3.911937 & 0.000000 & 3.266696 \\
\hline $\mathrm{H}$ & -2.190679 & 0.000000 & 3.706446 \\
\hline $\mathrm{C}$ & 2.660994 & -1.140683 & 1.843947 \\
\hline $\mathrm{C}$ & 2.660994 & 1.140683 & 1.843946 \\
\hline $\mathrm{C}$ & 2.891767 & 0.000000 & 2.869888 \\
\hline $\mathrm{C}$ & 3.660077 & 0.716456 & 0.780407 \\
\hline $\mathrm{C}$ & 3.660077 & -0.716456 & 0.780408 \\
\hline $\mathrm{C}$ & 4.450508 & -1.421885 & -0.085986 \\
\hline $\mathrm{C}$ & 4.450508 & 1.421885 & -0.085987 \\
\hline $\mathrm{C}$ & 5.301506 & -0.718348 & -0.991577 \\
\hline $\mathrm{C}$ & 5.301506 & 0.718348 & -0.991578 \\
\hline $\mathrm{C}$ & 6.159816 & -1.400232 & -1.893012 \\
\hline $\mathrm{C}$ & 6.159816 & 1.400232 & -1.893013 \\
\hline $\mathrm{C}$ & 6.980289 & -0.707306 & -2.756730 \\
\hline C & 6.980289 & 0.707306 & -2.756731 \\
\hline $\mathrm{C}$ & 1.269044 & 0.784904 & 1.240084 \\
\hline C & 1.269044 & -0.784904 & 1.240085 \\
\hline $\mathrm{H}$ & 4.458005 & -2.510402 & -0.092634 \\
\hline $\mathrm{H}$ & 4.458005 & 2.510402 & -0.092635 \\
\hline $\mathrm{H}$ & 6.159100 & -2.488236 & -1.890418 \\
\hline $\mathrm{H}$ & 6.159100 & 2.488236 & -1.890419 \\
\hline $\mathrm{H}$ & 7.630370 & 1.245278 & -3.441898 \\
\hline $\mathrm{H}$ & 7.630370 & -1.245278 & -3.441897 \\
\hline $\mathrm{H}$ & 2.763273 & -2.166402 & 2.206565 \\
\hline $\mathrm{H}$ & 2.763273 & 2.166402 & 2.206564 \\
\hline $\mathrm{H}$ & 3.911937 & 0.000000 & 3.266697 \\
\hline $\mathrm{H}$ & 2.190679 & 0.000000 & 3.706447 \\
\hline $\mathrm{H}$ & 0.668451 & 1.211913 & 2.002389 \\
\hline $\mathrm{H}$ & 0.668451 & -1.211913 & 2.002390 \\
\hline $\mathrm{H}$ & 1.132044 & -1.271389 & 0.296971 \\
\hline $\mathrm{H}$ & 1.132044 & 1.271389 & 0.296970 \\
\hline
\end{tabular}

DN6 N2-N1

$\begin{array}{lrrr}\text { C } & -3.587397 & -0.851012 & 0.716456 \\ \text { C } & -3.587397 & -0.851012 & -0.716456 \\ \text { C } & -4.373781 & 0.019057 & 1.421885\end{array}$




\begin{tabular}{|c|c|c|c|}
\hline C & -5.220549 & 0.928605 & 0.718348 \\
\hline $\mathrm{C}$ & -5.220549 & 0.928605 & -0.718348 \\
\hline C & -4.373781 & 0.019057 & -1.421885 \\
\hline $\mathrm{C}$ & -6.074648 & 1.834030 & 1.400232 \\
\hline $\mathrm{C}$ & -6.891086 & 2.701563 & 0.707306 \\
\hline C & -6.891086 & 2.701563 & -0.707306 \\
\hline C & -6.074648 & 1.834030 & -1.400232 \\
\hline C & -2.593283 & -1.919197 & 1.140683 \\
\hline$C$ & -2.593283 & -1.919197 & -1.140683 \\
\hline C & -2.828835 & -2.944052 & 0.000000 \\
\hline C & -1.198533 & -1.321829 & 0.784904 \\
\hline C & -1.198533 & -1.321829 & -0.784904 \\
\hline $\mathrm{H}$ & -4.381247 & 0.025740 & -2.510402 \\
\hline $\mathrm{H}$ & -4.381247 & 0.025740 & 2.510402 \\
\hline $\mathrm{H}$ & -6.073944 & 1.831433 & 2.488236 \\
\hline $\mathrm{H}$ & -7.537966 & 3.389753 & 1.245278 \\
\hline $\mathrm{H}$ & -7.537966 & 3.389753 & -1.245278 \\
\hline $\mathrm{H}$ & -6.073944 & 1.831433 & -2.488236 \\
\hline $\mathrm{H}$ & -2.697251 & -2.281334 & 2.166402 \\
\hline H & -2.697251 & -2.281334 & -2.166402 \\
\hline $\mathrm{H}$ & -3.850844 & -3.336100 & 0.000000 \\
\hline $\mathrm{H}$ & -1.053862 & -0.357522 & 1.282664 \\
\hline $\mathrm{H}$ & -1.053862 & -0.357522 & -1.282664 \\
\hline $\mathrm{H}$ & -2.131654 & -3.783868 & 0.000000 \\
\hline $\mathrm{H}$ & -0.333062 & -1.950818 & -0.800210 \\
\hline $\mathrm{H}$ & -0.333062 & -1.950818 & 0.800210 \\
\hline $\mathrm{C}$ & 2.728648 & -1.944003 & -1.140683 \\
\hline C & 2.728648 & -1.944003 & 1.140683 \\
\hline C & 2.954636 & -2.971010 & 0.000000 \\
\hline $\mathrm{H}$ & 2.829235 & -2.307094 & -2.166402 \\
\hline $\mathrm{H}$ & 2.829235 & -2.307094 & 2.166402 \\
\hline $\mathrm{H}$ & 3.972945 & -3.372569 & 0.000000 \\
\hline $\mathrm{H}$ & 2.249656 & -3.804291 & 0.000000 \\
\hline $\mathrm{C}$ & 3.732677 & -0.885133 & 0.716456 \\
\hline $\mathrm{C}$ & 3.732677 & -0.885133 & -0.716456 \\
\hline $\mathrm{C}$ & 4.527138 & -0.022432 & -1.421885 \\
\hline $\mathrm{C}$ & 4.527138 & -0.022432 & 1.421885 \\
\hline $\mathrm{C}$ & 5.382348 & 0.879182 & -0.718348 \\
\hline $\mathrm{C}$ & 5.382348 & 0.879182 & 0.718348 \\
\hline C & 6.244850 & 1.776607 & -1.400232 \\
\hline C & 6.244850 & 1.776607 & 1.400232 \\
\hline C & 7.069340 & 2.636491 & -0.707306 \\
\hline$C$ & 7.069340 & 2.636491 & 0.707306 \\
\hline
\end{tabular}




$\begin{array}{lrrr}\mathrm{H} & 4.534666 & -0.015819 & -2.510402 \\ \mathrm{H} & 4.534666 & -0.015819 & 2.510402 \\ \mathrm{H} & 6.244122 & 1.774016 & -2.488236 \\ \mathrm{H} & 6.244122 & 1.774016 & 2.488236 \\ \mathrm{H} & 7.722608 & 3.318620 & 1.245278 \\ \mathrm{H} & 7.722608 & 3.318620 & -1.245278 \\ \mathrm{H} & 1.774903 & -1.524953 & 0.896412 \\ \mathrm{H} & 1.774903 & -1.524953 & -0.896412\end{array}$

DN6 N1-N1

$\begin{array}{lrrr}\mathrm{C} & -3.660077 & 0.716456 & 0.965086 \\ \mathrm{C} & -3.660077 & -0.716456 & 0.965086 \\ \mathrm{C} & -4.450508 & 1.421885 & 0.098692 \\ \mathrm{C} & -5.301506 & 0.718348 & -0.806899 \\ \mathrm{C} & -5.301506 & -0.718348 & -0.806899 \\ \mathrm{C} & -4.450508 & -1.421885 & 0.098692 \\ \mathrm{C} & -6.159816 & 1.400232 & -1.708334 \\ \mathrm{C} & -6.980289 & 0.707306 & -2.572052 \\ \mathrm{C} & -6.980289 & -0.707306 & -2.572052 \\ \mathrm{C} & -6.159816 & -1.400232 & -1.708334 \\ \mathrm{C} & -2.660994 & 1.140683 & 2.028625 \\ \mathrm{C} & -2.660994 & -1.140683 & 2.028625 \\ \mathrm{C} & -2.891767 & 0.000000 & 3.054567 \\ \mathrm{H} & -1.705307 & -0.896412 & 1.614024 \\ \mathrm{H} & -1.705307 & 0.896412 & 1.614024 \\ \mathrm{H} & -4.458005 & -2.510402 & 0.092044 \\ \mathrm{H} & -4.458005 & 2.510402 & 0.092044 \\ \mathrm{H} & -6.159100 & 2.488236 & -1.705740 \\ \mathrm{H} & -7.630370 & 1.245278 & -3.257219 \\ \mathrm{H} & -7.630370 & -1.245278 & -3.257219 \\ \mathrm{H} & -6.159100 & -2.488236 & -1.705740 \\ \mathrm{H} & -2.763273 & 2.166402 & 2.391243 \\ \mathrm{H} & -2.763273 & -2.166402 & 2.391243 \\ \mathrm{H} & -3.911937 & 0.000000 & 3.451375 \\ \mathrm{H} & -2.190679 & 0.000000 & 3.891125 \\ \mathrm{C} & 2.660994 & -1.140683 & 2.028625 \\ \mathrm{C} & 2.660994 & 1.140683 & 2.028625 \\ \mathrm{C} & 2.891767 & 0.000000 & 3.054567 \\ \mathrm{H} & 2.763273 & -2.166402 & 2.391243 \\ \mathrm{H} & 2.763273 & 2.166402 & 2.391243 \\ \mathrm{C} & 3.911937 & 0.000000 & 3.451375 \\ & 2.190679 & 0.000000 & 3.891125 \\ \mathrm{H} & 3.660077 & 0.716456 & 0.965086\end{array}$




$\begin{array}{lrrr}\text { C } & 3.660077 & -0.716456 & 0.965086 \\ \text { C } & 4.450508 & -1.421885 & 0.098692 \\ \text { C } & 4.450508 & 1.421885 & 0.098692 \\ \text { C } & 5.301506 & -0.718348 & -0.806899 \\ \text { C } & 5.301506 & 0.718348 & -0.806899 \\ \text { C } & 6.159816 & -1.400232 & -1.708334 \\ \text { C } & 6.159816 & 1.400232 & -1.708334 \\ \text { C } & 6.980289 & -0.707306 & -2.572052 \\ \text { C } & 6.980289 & 0.707306 & -2.572052 \\ \text { H } & 4.458005 & -2.510402 & 0.092044 \\ \text { H } & 4.458005 & 2.510402 & 0.092044 \\ \text { H } & 6.159100 & -2.488236 & -1.705740 \\ \text { H } & 6.159100 & 2.488236 & -1.705740 \\ \text { H } & 7.630370 & 1.245278 & -3.257219 \\ \text { H } & 7.630370 & -1.245278 & -3.257219 \\ \text { H } & 1.705307 & -0.896412 & 1.614024 \\ \text { H } & 1.705307 & 0.896412 & 1.614024\end{array}$

DN6 NO-NO

$\begin{array}{lrrr}\text { C } & -3.660077 & 0.716456 & 1.767620 \\ \text { C } & -3.660077 & -0.716456 & 1.767620 \\ \text { C } & -4.450508 & 1.421885 & 0.901226 \\ \text { C } & -5.301506 & 0.718348 & -0.004365 \\ \text { C } & -5.301506 & -0.718348 & -0.004365 \\ \text { C } & -4.450508 & -1.421885 & 0.901226 \\ \text { C } & -6.159816 & 1.400232 & -0.905800 \\ \text { C } & -6.980289 & 0.707306 & -1.769518 \\ \text { C } & -6.980289 & -0.707306 & -1.769518 \\ \text { C } & -6.159816 & -1.400232 & -0.905800 \\ \text { H } & -4.458005 & -2.510402 & 0.894578 \\ \text { H } & -4.458005 & 2.510402 & 0.894578 \\ \text { H } & -6.159100 & 2.488236 & -0.903206 \\ \text { H } & -7.630370 & 1.245278 & -2.454685 \\ \text { H } & -7.630370 & -1.245278 & -2.454685 \\ \text { H } & -6.159100 & -2.488236 & -0.903206 \\ \text { H } & -2.945450 & 1.019898 & 2.528351 \\ \text { H } & -2.945450 & -1.019898 & 2.528351 \\ \text { C } & 3.660077 & 0.716456 & 1.767620 \\ \text { C } & 3.660077 & -0.716456 & 1.767620 \\ \text { C } & 4.450508 & -1.421885 & 0.901226 \\ \text { C } & 4.450508 & 1.421885 & 0.901226 \\ \text { C } & 5.301506 & -0.718348 & -0.004365 \\ \text { C } & 5.301506 & 0.718348 & -0.004365\end{array}$




$\begin{array}{lrrr}\text { C } & 6.159816 & -1.400232 & -0.905800 \\ \mathrm{C} & 6.159816 & 1.400232 & -0.905800 \\ \mathrm{C} & 6.980289 & -0.707306 & -1.769518 \\ \mathrm{C} & 6.980289 & 0.707306 & -1.769518 \\ \mathrm{H} & 4.458005 & -2.510402 & 0.894578 \\ \mathrm{H} & 4.458005 & 2.510402 & 0.894578 \\ \mathrm{H} & 6.159100 & -2.488236 & -0.903206 \\ \mathrm{H} & 6.159100 & 2.488236 & -0.903206 \\ \mathrm{H} & 7.630370 & 1.245278 & -2.454685 \\ \mathrm{H} & 7.630370 & -1.245278 & -2.454685 \\ \mathrm{H} & 2.945450 & 1.019898 & 2.528351 \\ \mathrm{H} & 2.945450 & -1.019898 & 2.528351\end{array}$

A6N Full

\begin{tabular}{|c|c|c|c|}
\hline C & -4.554393 & -0.047028 & 0.716328 \\
\hline $\mathrm{C}$ & -4.554393 & -0.047028 & -0.716328 \\
\hline $\mathrm{C}$ & -5.099406 & 0.992148 & 1.421114 \\
\hline $\mathrm{C}$ & -5.688939 & 2.086354 & 0.718489 \\
\hline $\mathrm{C}$ & -5.688939 & 2.086354 & -0.718489 \\
\hline $\mathrm{C}$ & -5.099406 & 0.992148 & -1.421114 \\
\hline $\mathrm{C}$ & -6.271004 & 3.186934 & 1.399799 \\
\hline $\mathrm{C}$ & -6.821566 & 4.243524 & 0.707393 \\
\hline $\mathrm{C}$ & -6.821566 & 4.243524 & -0.707393 \\
\hline $\mathrm{C}$ & -6.271004 & 3.186934 & -1.399799 \\
\hline $\mathrm{C}$ & -3.811543 & -1.302859 & 1.140659 \\
\hline $\mathrm{C}$ & -3.811543 & -1.302859 & -1.140659 \\
\hline $\mathrm{C}$ & -4.250154 & -2.259243 & 0.000000 \\
\hline $\mathrm{C}$ & -2.323060 & -1.002123 & 0.785469 \\
\hline $\mathrm{C}$ & -2.323060 & -1.002123 & -0.785469 \\
\hline $\mathrm{H}$ & -5.097140 & 1.004936 & -2.509721 \\
\hline $\mathrm{H}$ & -5.097140 & 1.004936 & 2.509721 \\
\hline $\mathrm{H}$ & -6.269495 & 3.184814 & 2.487904 \\
\hline $\mathrm{H}$ & -7.255696 & 5.081714 & 1.245229 \\
\hline $\mathrm{H}$ & -7.255696 & 5.081714 & -1.245229 \\
\hline $\mathrm{H}$ & -6.269495 & 3.184814 & -2.487904 \\
\hline $\mathrm{H}$ & -3.985197 & -1.637961 & 2.166432 \\
\hline $\mathrm{H}$ & -3.985197 & -1.637961 & -2.166432 \\
\hline $\mathrm{H}$ & -5.330279 & -2.438190 & 0.000000 \\
\hline $\mathrm{H}$ & -1.986635 & -0.089525 & 1.287517 \\
\hline $\mathrm{H}$ & -1.986635 & -0.089525 & -1.287517 \\
\hline $\mathrm{H}$ & -3.734889 & -3.221626 & 0.000000 \\
\hline $\mathrm{C}$ & -1.267044 & -2.153480 & -0.806062 \\
\hline $\mathrm{C}$ & -1.267044 & -2.153480 & 0.80606 \\
\hline
\end{tabular}




\begin{tabular}{|c|c|c|c|}
\hline C & -1.504793 & -3.317025 & -1.756294 \\
\hline C & -1.504793 & -3.317025 & 1.756294 \\
\hline C & 1.403824 & -2.369345 & -1.142009 \\
\hline $\mathrm{C}$ & 1.403824 & -2.369345 & 1.142009 \\
\hline $\mathrm{C}$ & 1.427204 & -3.421205 & 0.000000 \\
\hline $\mathrm{C}$ & 0.155641 & -1.506434 & 0.785626 \\
\hline $\mathrm{C}$ & 0.155641 & -1.506434 & -0.785626 \\
\hline $\mathrm{H}$ & -0.770600 & -4.119811 & -1.633106 \\
\hline $\mathrm{H}$ & -1.431849 & -2.968143 & -2.795529 \\
\hline $\mathrm{H}$ & -2.497240 & -3.761838 & -1.635737 \\
\hline $\mathrm{H}$ & -0.770600 & -4.119811 & 1.633106 \\
\hline $\mathrm{H}$ & -2.497240 & -3.761838 & 1.635737 \\
\hline $\mathrm{H}$ & -1.431849 & -2.968143 & 2.795529 \\
\hline $\mathrm{H}$ & 1.430946 & -2.745827 & -2.167506 \\
\hline $\mathrm{H}$ & 1.430946 & -2.745827 & 2.167506 \\
\hline $\mathrm{H}$ & 2.342539 & -4.021596 & 0.000000 \\
\hline $\mathrm{H}$ & 0.565107 & -4.089747 & 0.000000 \\
\hline $\mathrm{H}$ & 0.207042 & -0.534683 & 1.286729 \\
\hline $\mathrm{H}$ & 0.207042 & -0.534683 & -1.286729 \\
\hline $\mathrm{C}$ & 2.585685 & -1.512931 & 0.721251 \\
\hline $\mathrm{C}$ & 2.585685 & -1.512931 & -0.721251 \\
\hline C & 3.501040 & -0.790774 & -1.426217 \\
\hline $\mathrm{C}$ & 3.501040 & -0.790774 & 1.426217 \\
\hline C & 4.490170 & -0.020401 & -0.724872 \\
\hline $\mathrm{C}$ & 4.490170 & -0.020401 & 0.724872 \\
\hline $\mathrm{C}$ & 5.448930 & 0.740760 & -1.401268 \\
\hline $\mathrm{C}$ & 5.448930 & 0.740760 & 1.401268 \\
\hline $\mathrm{C}$ & 6.402735 & 1.512001 & -0.721895 \\
\hline $\mathrm{C}$ & 7.374431 & 2.305015 & -1.406451 \\
\hline C & 7.374431 & 2.305015 & 1.406451 \\
\hline C & 8.288301 & 3.055030 & -0.712718 \\
\hline $\mathrm{C}$ & 6.402735 & 1.512001 & 0.721895 \\
\hline $\mathrm{C}$ & 8.288301 & 3.055030 & 0.712718 \\
\hline $\mathrm{H}$ & 3.505806 & -0.781591 & -2.514668 \\
\hline $\mathrm{H}$ & 3.505806 & -0.781591 & 2.514668 \\
\hline $\mathrm{H}$ & 5.449168 & 0.741393 & -2.490011 \\
\hline $\mathrm{H}$ & 5.449168 & 0.741393 & 2.490011 \\
\hline $\mathrm{H}$ & 7.372484 & 2.303524 & -2.494355 \\
\hline $\mathrm{H}$ & 7.372484 & 2.303524 & 2.494355 \\
\hline $\mathrm{H}$ & 9.021965 & 3.653730 & -1.245979 \\
\hline $\mathrm{H}$ & 9.021965 & 3.653730 & 1.24597 \\
\hline
\end{tabular}

A6N N2-A2 


\begin{tabular}{|c|c|c|c|}
\hline C & -4.703896 & -0.517737 & 0.716328 \\
\hline $\mathrm{C}$ & -4.703896 & -0.517737 & -0.716328 \\
\hline C & -5.277007 & 0.506210 & 1.421114 \\
\hline C & -5.896120 & 1.583955 & 0.718489 \\
\hline $\mathrm{C}$ & -5.896120 & 1.583955 & -0.718489 \\
\hline C & -5.277007 & 0.506210 & -1.421114 \\
\hline C & -6.507942 & 2.668276 & 1.399799 \\
\hline$C$ & -7.087074 & 3.709480 & 0.707393 \\
\hline$C$ & -7.087074 & 3.709480 & -0.707393 \\
\hline C & -6.507942 & 2.668276 & -1.399799 \\
\hline C & -3.927120 & -1.752873 & 1.140659 \\
\hline C & -3.927120 & -1.752873 & -1.140659 \\
\hline $\mathrm{C}$ & -4.339523 & -2.720847 & 0.000000 \\
\hline C & -2.447380 & -1.411712 & 0.785469 \\
\hline C & -2.447380 & -1.411712 & -0.785469 \\
\hline $\mathrm{H}$ & -1.705525 & -2.092090 & -1.148287 \\
\hline $\mathrm{H}$ & -1.705525 & -2.092090 & 1.148287 \\
\hline $\mathrm{H}$ & -2.142922 & -0.510968 & -1.276250 \\
\hline $\mathrm{H}$ & -2.142922 & -0.510968 & 1.276250 \\
\hline $\mathrm{H}$ & -5.275090 & 0.519055 & -2.509721 \\
\hline $\mathrm{H}$ & -5.275090 & 0.519055 & 2.509721 \\
\hline $\mathrm{H}$ & -6.506375 & 2.666197 & 2.487904 \\
\hline $\mathrm{H}$ & -7.543870 & 4.535536 & 1.245229 \\
\hline $\mathrm{H}$ & -7.543870 & 4.535536 & -1.245229 \\
\hline $\mathrm{H}$ & -6.506375 & 2.666197 & -2.487904 \\
\hline $\mathrm{H}$ & -4.091585 & -2.092579 & 2.166432 \\
\hline $\mathrm{H}$ & -4.091585 & -2.092579 & -2.166432 \\
\hline $\mathrm{H}$ & -5.414375 & -2.929143 & 0.000000 \\
\hline $\mathrm{H}$ & -3.798241 & -3.668840 & 0.000000 \\
\hline $\mathrm{C}$ & 1.315355 & -2.676932 & -1.142009 \\
\hline $\mathrm{C}$ & 1.315355 & -2.676932 & 1.142009 \\
\hline $\mathrm{C}$ & 1.367373 & -3.727764 & 0.000000 \\
\hline C & 2.473456 & -1.788649 & 0.721251 \\
\hline $\mathrm{C}$ & 2.473456 & -1.788649 & -0.721251 \\
\hline $\mathrm{C}$ & 3.368805 & -1.041831 & -1.426217 \\
\hline $\mathrm{C}$ & 3.368805 & -1.041831 & 1.426217 \\
\hline $\mathrm{C}$ & 4.336588 & -0.244807 & -0.724872 \\
\hline $\mathrm{C}$ & 4.336588 & -0.244807 & 0.724872 \\
\hline $\mathrm{C}$ & 5.274263 & 0.542182 & -1.401268 \\
\hline $\mathrm{C}$ & 5.274263 & 0.542182 & 1.401268 \\
\hline $\mathrm{C}$ & 6.206711 & 1.339112 & -0.721895 \\
\hline $\mathrm{C}$ & 7.156450 & 2.158294 & -1.406451 \\
\hline $\mathrm{C}$ & 7.156450 & 2.158294 & 1.406451 \\
\hline
\end{tabular}




$\begin{array}{lrrr}\text { C } & 8.049556 & 2.932919 & -0.712718 \\ \text { C } & 6.206711 & 1.339112 & 0.721895 \\ \text { C } & 8.049556 & 2.932919 & 0.712718 \\ \text { C } & 0.044136 & -1.848333 & 0.785626 \\ \text { C } & 0.044136 & -1.848333 & -0.785626 \\ \text { H } & -0.886027 & -2.232912 & -1.148664 \\ \text { H } & -0.886027 & -2.232912 & 1.148664 \\ \text { H } & 0.068495 & -0.897364 & -1.275488 \\ \text { H } & 0.068495 & -0.897364 & 1.275488 \\ \text { H } & 3.373319 & -1.032522 & -2.514668 \\ \text { H } & 3.373319 & -1.032522 & 2.514668 \\ \text { H } & 5.274483 & 0.542821 & -2.490011 \\ \text { H } & 5.274483 & 0.542821 & 2.490011 \\ \text { H } & 7.154545 & 2.156752 & -2.494355 \\ \text { H } & 7.154545 & 2.156752 & 2.494355 \\ \text { H } & 8.766643 & 3.551378 & -1.245979 \\ \text { H } & 8.766643 & 3.551378 & 1.245979 \\ \text { H } & 1.352721 & -3.052535 & -2.167506 \\ \text { H } & 1.352721 & -3.052535 & 2.167506 \\ \text { H } & 2.298720 & -4.303005 & 0.000000 \\ \text { H } & 0.523803 & -4.419537 & 0.000000\end{array}$

A6N N2-A1

$\begin{array}{lrrr}\text { C } & -4.712303 & -0.632649 & 0.716328 \\ \text { C } & -4.712303 & -0.632649 & -0.716328 \\ \text { C } & -5.286457 & 0.390714 & 1.421114 \\ \text { C } & -5.906668 & 1.467828 & 0.718489 \\ \text { C } & -5.906668 & 1.467828 & -0.718489 \\ \text { C } & -5.286457 & 0.390714 & -1.421114 \\ \text { C } & -6.519594 & 2.551525 & 1.399799 \\ \text { C } & -7.099787 & 3.592138 & 0.707393 \\ \text { C } & -7.099787 & 3.592138 & -0.707393 \\ \text { C } & -6.519594 & 2.551525 & -1.399799 \\ \text { C } & -3.934269 & -1.866993 & 1.140659 \\ \text { C } & -3.934269 & -1.866993 & -1.140659 \\ \text { C } & -4.345686 & -2.835386 & 0.000000 \\ \text { C } & -2.454878 & -1.524324 & 0.785469 \\ \text { C } & -2.454878 & -1.524324 & -0.785469 \\ \text { H } & -1.712330 & -2.203946 & -1.148287 \\ \text { H } & -1.712330 & -2.203946 & 1.148287 \\ \text { H } & -2.151337 & -0.623270 & -1.276250 \\ \text { H } & -2.151337 & -0.623270 & 1.276250 \\ \text { H } & -5.284553 & 0.403561 & -2.509721\end{array}$




\begin{tabular}{|c|c|c|c|}
\hline $\mathrm{H}$ & -5.284553 & 0.403561 & 2.509721 \\
\hline $\mathrm{H}$ & -6.518025 & 2.549447 & 2.487904 \\
\hline $\mathrm{H}$ & -7.557424 & 4.417728 & 1.245229 \\
\hline $\mathrm{H}$ & -7.557424 & 4.417728 & -1.245229 \\
\hline $\mathrm{H}$ & -6.518025 & 2.549447 & -2.487904 \\
\hline $\mathrm{H}$ & -4.098388 & -2.206866 & 2.166432 \\
\hline $\mathrm{H}$ & -4.098388 & -2.206866 & -2.166432 \\
\hline $\mathrm{H}$ & -5.420325 & -3.044777 & 0.000000 \\
\hline $\mathrm{H}$ & -3.803438 & -3.782827 & 0.000000 \\
\hline $\mathrm{C}$ & 2.466340 & -1.896248 & 0.721251 \\
\hline $\mathrm{C}$ & 2.466340 & -1.896248 & -0.721251 \\
\hline $\mathrm{C}$ & 3.360928 & -1.148518 & -1.426217 \\
\hline C & 3.360928 & -1.148518 & 1.426217 \\
\hline $\mathrm{C}$ & 4.327898 & -0.350508 & -0.724872 \\
\hline $\mathrm{C}$ & 4.327898 & -0.350508 & 0.724872 \\
\hline $\mathrm{C}$ & 5.264771 & 0.437436 & -1.401268 \\
\hline $\mathrm{C}$ & 5.264771 & 0.437436 & 1.401268 \\
\hline $\mathrm{C}$ & 6.196406 & 1.235315 & -0.721895 \\
\hline $\mathrm{C}$ & 7.145310 & 2.055464 & -1.406451 \\
\hline $\mathrm{C}$ & 7.145310 & 2.055464 & 1.406451 \\
\hline $\mathrm{C}$ & 8.037627 & 2.830999 & -0.712718 \\
\hline $\mathrm{C}$ & 6.196406 & 1.235315 & 0.721895 \\
\hline $\mathrm{C}$ & 8.037627 & 2.830999 & 0.712718 \\
\hline $\mathrm{C}$ & 1.309144 & -2.785710 & -1.142009 \\
\hline $\mathrm{C}$ & 1.309144 & -2.785710 & 1.142009 \\
\hline $\mathrm{C}$ & 1.362233 & -3.836489 & 0.000000 \\
\hline $\mathrm{H}$ & 1.346893 & -3.161275 & -2.167506 \\
\hline $\mathrm{H}$ & 1.346893 & -3.161275 & 2.167506 \\
\hline $\mathrm{H}$ & 2.294166 & -4.410780 & 0.000000 \\
\hline $\mathrm{H}$ & 0.519368 & -4.529121 & 0.000000 \\
\hline $\mathrm{H}$ & 3.365432 & -1.139204 & -2.514668 \\
\hline $\mathrm{H}$ & 3.365432 & -1.139204 & 2.514668 \\
\hline $\mathrm{H}$ & 5.264990 & 0.438075 & -2.490011 \\
\hline $\mathrm{H}$ & 5.264990 & 0.438075 & 2.490011 \\
\hline $\mathrm{H}$ & 7.143407 & 2.053920 & -2.494355 \\
\hline $\mathrm{H}$ & 7.143407 & 2.053920 & 2.494355 \\
\hline $\mathrm{H}$ & 8.754083 & 3.450188 & -1.245979 \\
\hline $\mathrm{H}$ & 8.754083 & 3.450188 & 1.245979 \\
\hline $\mathrm{H}$ & 0.435919 & -2.217796 & -0.897365 \\
\hline $\mathrm{H}$ & 0.435919 & -2.217796 & 0.897365 \\
\hline
\end{tabular}

A6N N1-A2

$\begin{array}{llll}\text { C } & -4.840020 & -0.650020 & 0.716328\end{array}$ 


\begin{tabular}{|c|c|c|c|}
\hline $\mathrm{C}$ & -4.840020 & -0.650020 & -0.716328 \\
\hline $\mathrm{C}$ & -5.422710 & 0.368507 & 1.421114 \\
\hline C & -6.051904 & 1.440397 & 0.718489 \\
\hline C & -6.051904 & 1.440397 & -0.718489 \\
\hline C & -5.422710 & 0.368507 & -1.421114 \\
\hline C & -6.673869 & 2.518932 & 1.399799 \\
\hline $\mathrm{C}$ & -7.262741 & 3.554659 & 0.707393 \\
\hline C & -7.262741 & 3.554659 & -0.707393 \\
\hline $\mathrm{C}$ & -6.673869 & 2.518932 & -1.399799 \\
\hline C & -4.051694 & -1.877816 & 1.140659 \\
\hline $\mathrm{C}$ & -4.051694 & -1.877816 & -1.140659 \\
\hline C & -4.455000 & -2.849616 & 0.000000 \\
\hline $\mathrm{H}$ & -5.420913 & 0.381369 & -2.509721 \\
\hline $\mathrm{H}$ & -5.420913 & 0.381369 & 2.509721 \\
\hline $\mathrm{H}$ & -6.672282 & 2.516868 & 2.487904 \\
\hline $\mathrm{H}$ & -7.727264 & 4.376394 & 1.245229 \\
\hline $\mathrm{H}$ & -7.727264 & 4.376394 & -1.245229 \\
\hline $\mathrm{H}$ & -6.672282 & 2.516868 & -2.487904 \\
\hline $\mathrm{H}$ & -4.212965 & -2.219050 & 2.166432 \\
\hline $\mathrm{H}$ & -4.212965 & -2.219050 & -2.166432 \\
\hline $\mathrm{H}$ & -5.527851 & -3.067984 & 0.000000 \\
\hline $\mathrm{H}$ & -3.904850 & -3.792490 & 0.000000 \\
\hline $\mathrm{H}$ & -3.038688 & -1.634235 & -0.896964 \\
\hline $\mathrm{H}$ & -3.038688 & -1.634235 & 0.896964 \\
\hline C & 2.348936 & -1.853559 & 0.721251 \\
\hline $\mathrm{C}$ & 2.348936 & -1.853559 & -0.721251 \\
\hline $\mathrm{C}$ & 3.237241 & -1.098376 & -1.426217 \\
\hline C & 3.237241 & -1.098376 & 1.426217 \\
\hline C & 4.197506 & -0.292310 & -0.724872 \\
\hline $\mathrm{C}$ & 4.197506 & -0.292310 & 0.724872 \\
\hline $\mathrm{C}$ & 5.127759 & 0.503439 & -1.401268 \\
\hline $\mathrm{C}$ & 5.127759 & 0.503439 & 1.401268 \\
\hline $\mathrm{C}$ & 6.052691 & 1.309079 & -0.721895 \\
\hline $\mathrm{C}$ & 6.994705 & 2.137133 & -1.406451 \\
\hline $\mathrm{C}$ & 6.994705 & 2.137133 & 1.406451 \\
\hline $\mathrm{C}$ & 7.880507 & 2.920100 & -0.712718 \\
\hline $\mathrm{C}$ & 6.052691 & 1.309079 & 0.721895 \\
\hline $\mathrm{C}$ & 7.880507 & 2.920100 & 0.712718 \\
\hline $\mathrm{C}$ & -0.079717 & -1.936025 & 0.785626 \\
\hline C & -0.079717 & -1.936025 & -0.785626 \\
\hline l & 1.199218 & -2.752665 & -1.142009 \\
\hline $\mathrm{C}$ & 1.199218 & -2.752665 & 1.142009 \\
\hline $\mathrm{C}$ & 1.261089 & -3.802963 & 0.000000 \\
\hline
\end{tabular}




$\begin{array}{lrrr}\mathrm{H} & 1.240105 & -3.127901 & -2.167506 \\ \mathrm{H} & 1.240105 & -3.127901 & 2.167506 \\ \mathrm{H} & 2.197790 & -4.369443 & 0.000000 \\ \mathrm{H} & 0.424044 & -4.502617 & 0.000000 \\ \mathrm{H} & -1.006232 & -2.329311 & -1.148664 \\ \mathrm{H} & -1.006232 & -2.329311 & 1.148664 \\ \mathrm{H} & -0.064278 & -0.984870 & -1.275488 \\ \mathrm{H} & -0.064278 & -0.984870 & 1.275488 \\ \mathrm{H} & 3.241668 & -1.089025 & -2.514668 \\ \mathrm{H} & 3.241668 & -1.089025 & 2.514668 \\ \mathrm{H} & 5.127973 & 0.504080 & -2.490011 \\ \mathrm{H} & 5.127973 & 0.504080 & 2.490011 \\ \mathrm{H} & 6.992815 & 2.135573 & -2.494355 \\ \mathrm{H} & 6.992815 & 2.135573 & 2.494355 \\ \mathrm{H} & 8.591762 & 3.545258 & -1.245979 \\ \mathrm{H} & 8.591762 & 3.545258 & 1.245979\end{array}$

$\begin{array}{rrrr}\text { A6N } & \text { N1-A1 } & & \\ \text { C } & -4.856820 & -0.782494 & 0.716328 \\ \text { C } & -4.856820 & -0.782494 & -0.716328 \\ \text { C } & -5.441319 & 0.234996 & 1.421114 \\ \text { C } & -6.072419 & 1.305767 & 0.718489 \\ \text { C } & -6.072419 & 1.305767 & -0.718489 \\ \text { C } & -5.441319 & 0.234996 & -1.421114 \\ \text { C } & -6.696300 & 2.383194 & 1.399799 \\ \text { C } & -7.287012 & 3.417872 & 0.707393 \\ \text { C } & -7.287012 & 3.417872 & -0.707393 \\ \text { C } & -6.696300 & 2.383194 & -1.399799 \\ \text { C } & -4.066314 & -2.008886 & 1.140659 \\ \text { C } & -4.066314 & -2.008886 & -1.140659 \\ \text { C } & -4.467891 & -2.981401 & 0.000000 \\ \text { H } & -3.032204 & -1.758285 & -0.891780 \\ \text { H } & -3.032204 & -1.758285 & 0.891780 \\ \text { H } & -5.439546 & 0.247862 & -2.509721 \\ \text { H } & -5.439546 & 0.247862 & 2.509721 \\ \text { H } & -6.694711 & 2.381133 & 2.487904 \\ \text { H } & -7.752995 & 4.238781 & 1.245229 \\ \text { H } & -7.752995 & 4.238781 & -1.245229 \\ \text { H } & -6.694711 & 2.381133 & -2.487904 \\ \text { H } & -4.226978 & -2.350407 & 2.166432 \\ \text { H } & -4.226978 & -2.350407 & -2.166432 \\ \text { H } & -5.540352 & -3.201677 & 0.000000 \\ \text { H } & -3.916066 & -3.923297 & 0.000000\end{array}$




$\begin{array}{lrrr}\mathrm{C} & 1.186146 & -2.874400 & -1.142009 \\ \mathrm{C} & 1.186146 & -2.874400 & 1.142009 \\ \mathrm{C} & 1.249883 & -3.924585 & 0.000000 \\ \mathrm{C} & 2.334263 & -1.973250 & 0.721251 \\ \mathrm{C} & 2.334263 & -1.973250 & -0.721251 \\ \mathrm{C} & 3.221225 & -1.216491 & -1.426217 \\ \mathrm{C} & 3.221225 & -1.216491 & 1.426217 \\ \mathrm{C} & 4.180056 & -0.408719 & -0.724872 \\ \mathrm{C} & 4.180056 & -0.408719 & 0.724872 \\ \mathrm{C} & 5.108891 & 0.388682 & -1.401268 \\ \mathrm{C} & 5.108891 & 0.388682 & 1.401268 \\ \mathrm{C} & 6.032391 & 1.195966 & -0.721895 \\ \mathrm{C} & 6.972931 & 2.025693 & -1.406451 \\ \mathrm{C} & 6.972931 & 2.025693 & 1.406451 \\ \mathrm{C} & 7.857340 & 2.810234 & -0.712718 \\ \mathrm{C} & 6.032391 & 1.195966 & 0.721895 \\ \mathrm{C} & 7.857340 & 2.810234 & 0.712718 \\ \mathrm{H} & 3.225635 & -1.207133 & -2.514668 \\ \mathrm{H} & 3.225635 & -1.207133 & 2.514668 \\ \mathrm{H} & 5.109105 & 0.389324 & -2.490011 \\ \mathrm{H} & 5.109105 & 0.389324 & 2.490011 \\ \mathrm{H} & 6.971043 & 2.024129 & -2.494355 \\ \mathrm{H} & 6.971043 & 2.024129 & 2.494355 \\ \mathrm{H} & 8.567482 & 3.436654 & -1.245979 \\ \mathrm{H} & 8.567482 & 3.436654 & 1.245979 \\ \mathrm{H} & 0.288512 & -2.303476 & -0.892161 \\ \mathrm{H} & 0.288512 & -2.303476 & 0.892161 \\ \mathrm{H} & 1.227699 & -3.249562 & -2.167506 \\ \mathrm{H} & 1.227699 & -3.249562 & 2.167506 \\ \mathrm{H} & 2.187590 & -4.489401 & 0.000000 \\ & 0.414083 & -4.625727 & 0.000000\end{array}$

A6N NO-AO

$\begin{array}{lrrr}\text { C } & -5.200858 & -1.780101 & 0.716328 \\ \text { C } & -5.200858 & -1.780101 & -0.716328 \\ \text { C } & -5.825995 & -0.787061 & 1.421114 \\ \text { C } & -6.499846 & 0.257334 & 0.718489 \\ \text { C } & -6.499846 & 0.257334 & -0.718489 \\ \text { C } & -5.825995 & -0.787061 & -1.421114 \\ \text { C } & -7.166754 & 1.308672 & 1.399799 \\ \text { C } & -7.798794 & 2.318635 & 0.707393 \\ \text { C } & -7.798794 & 2.318635 & -0.707393 \\ \text { C } & -7.166754 & 1.308672 & -1.399799\end{array}$




\begin{tabular}{lrrr}
$\mathrm{H}$ & -5.824742 & -0.774134 & -2.509721 \\
$\mathrm{H}$ & -5.824742 & -0.774134 & 2.509721 \\
$\mathrm{H}$ & -7.165083 & 1.306677 & 2.487904 \\
$\mathrm{H}$ & -8.297568 & 3.120044 & 1.245229 \\
$\mathrm{H}$ & -8.297568 & 3.120044 & -1.245229 \\
$\mathrm{H}$ & -7.165083 & 1.306677 & -2.487904 \\
$\mathrm{H}$ & -4.657802 & -2.552194 & -1.254166 \\
$\mathrm{H}$ & -4.657802 & -2.552194 & 1.254166 \\
$\mathrm{C}$ & 2.032469 & -2.679306 & 0.721251 \\
$\mathrm{C}$ & 2.032469 & -2.679306 & -0.721251 \\
$\mathrm{C}$ & 2.888127 & -1.887324 & -1.426217 \\
$\mathrm{C}$ & 2.888127 & -1.887324 & 1.426217 \\
$\mathrm{C}$ & 3.813534 & -1.041466 & -0.724872 \\
$\mathrm{C}$ & 3.813534 & -1.041466 & 0.724872 \\
$\mathrm{C}$ & 4.709390 & -0.207184 & -1.401268 \\
$\mathrm{C}$ & 4.709390 & -0.207184 & 1.401268 \\
$\mathrm{C}$ & 5.599514 & 0.636757 & -0.721895 \\
$\mathrm{C}$ & 6.505758 & 1.503813 & -1.406451 \\
$\mathrm{C}$ & 6.505758 & 1.503813 & 1.406451 \\
$\mathrm{C}$ & 7.357743 & 2.323449 & -0.712718 \\
$\mathrm{C}$ & 5.599514 & 0.636757 & 0.721895 \\
$\mathrm{C}$ & 7.357743 & 2.323449 & 0.712718 \\
$\mathrm{H}$ & 2.892155 & -1.877794 & -2.514668 \\
$\mathrm{H}$ & 2.892155 & -1.877794 & 2.514668 \\
$\mathrm{H}$ & 4.709577 & -0.206534 & -2.490011 \\
$\mathrm{H}$ & 4.709577 & -0.206534 & 2.490011 \\
$\mathrm{H}$ & 6.503935 & 1.502173 & -2.494355 \\
$\mathrm{H}$ & 6.503935 & 1.502173 & 2.494355 \\
$\mathrm{H}$ & 8.041992 & 2.978053 & -1.245979 \\
& 8.041992 & 2.978053 & 1.245979 \\
$\mathrm{H}$ & 1.311806 & -3.293592 & -1.254511 \\
\hline & 1.311806 & -3.293592 & 1.254511
\end{tabular}

